J

\title{
Influence of a 6-week arm exercise program on walking ability and health status after hip arthroplasty: A 1-year follow-up pilot study
}

\author{
Jérôme Maire, PhD; ${ }^{1}$ Benoit Dugué, PhD $;^{1-3 *}$ Anne-Françoise Faillenet-Maire, $\mathrm{MD} ;{ }^{4}$ Juhani Smolander, PhD; $^{5}$ \\ Nicolas Tordi, PhD; ${ }^{1}$ Bernard Parratte, PhD; ${ }^{6}$ Céline Grange, PhD; ${ }^{1}$ Jean-Denis Rouillon, MD, PhD $^{1}$ \\ ${ }^{1}$ Laboratoire des Sciences du Sport, Université de Franche-Comté, Besançon, France; ${ }^{2}$ Laboratory of Exercise- \\ Induced Physiological Adaptations (EA 3813), University of Poitiers, Poitiers, France; ${ }^{3}$ Minerva Foundation Institute \\ for Medical Research, Helsinki, Finland; ${ }^{4}$ Centre de Réadaptation Fonctionnelle, Service de Rééducation Gériatrique, \\ Quingey, France; ${ }^{5}$ ORTON Orthopaedic Hospital, Foundation for Invalids, Helsinki, Finland; ${ }^{6}$ Service d'Exploration \\ Fonctionnelle Neuro-Musculaire, Centre Hospitalier Universitaire, Besançon, France
}

\begin{abstract}
The influence of an upper-limb interval-training program after total hip arthroplasty (THA) in elderly patients was studied during a 1 yr follow-up on health status and walking ability. After surgery, 14 patients were randomly assigned to the control group that started a $6 \mathrm{wk}$ general rehabilitation program or the training group that combined it with an interval exercise program on an arm ergometer. A Western Ontario and McMaster Universities (WOMAC) Osteoarthritis Index was completed 1 mo before and 2 mo and 1 yr after surgery. A 6 min walking test was performed at 2 mo and 1 yr after surgery. The training group covered a significantly longer distance than the control group in the 6 min walk test and obtained significantly lower WOMAC scores both at 2 mo and 1 yr after surgery. However, both groups significantly improved in WOMAC and in their performance during the survey. Endurance-type upper-body aerobic training in a rehabilitation program might be important after THA.
\end{abstract}

Key words: 6 min walking test, arm exercise, elderly, exercise training, health status, hip arthroplasty, recovery after surgery, rehabilitation, transfer effect, WOMAC Osteoarthritis Index.

\section{INTRODUCTION}

Effective reconditioning programs that include endurance-type physical training have usually been prescribed for elderly patients after both cardiac and thoracic surgeries [1]. Such strategies have been shown to be suc- cessful in improving overall fitness and protecting elderly patients against a number of geriatric chronic diseases [2]. However, after total hip arthroplasty (THA), traditional daily rehabilitation programs consist of exercises for muscular strength, range of motion, aquatics, and walking. Although several authors have found positive effects of such rehabilitation programs [3-4], recent investigations have focused on new kinds of strength and postural stability exercises [5] and treadmill training with partial weight support [6]. These programs have been found to be more effective than the traditional rehabilitation programs.

Surprisingly, few investigators have considered endurance-type physical training in the rehabilitation

\footnotetext{
Abbreviations: $\mathrm{CG}=$ control group, $\mathrm{MMSE}=$ Mini-Mental State Examination, OA = osteoarthritis, SWEET = Square Wave Endurance Exercise Test, TG = training group, THA = total hip arthroplasty, WOMAC = Western Ontario and McMaster Universities (Osteoarthritis Index).

* Address all correspondence to Benoit Dugué, PhD; UFR Sciences du Sport de l'Université de Poitiers et Laboratoire des Adaptations Physiologiques aux Activités Physiques (EA 3813), 4 allée Jean Monnet, 86000, Poitiers, France; +33-54-945-4040; fax: +33-54-945-3396.

Email: benoit.dugue@univ-poitiers.fr

DOI: 10.1682/JRRD.2005.03.0058
} 
process after THA. We recently reported that a 6-week upper-limb interval training starting early after THA had positive effects in the recovery of elderly patients with osteoarthritis (OA) [7-8]. The positive effects of this aerobic training were observed 2 months after surgery on cardiorespiratory fitness, subjective health status (Western Ontario and McMaster University [WOMAC]), and walking ability THA. Moreover, we also reported that during such training, patients improved their ability to perceive exercise intensity and physical exertion [9]. Such improvements are important for THA patients in their everyday lives.

Our hypothesis is that a brief upper-limb exercise program would improve postoperative THA patients' endurance and physical fitness during and after rehabilitation. Whether the advantage observed 2 months after THA from upper-limb endurance training will have some significance later after the surgery is not clear. Therefore, this study examines the effect of our arm exercise program on walking ability and condition-specific WOMAC scores in our patients 1 year after THA. This upper-limb interval-training program was adapted from the Square Wave Endurance Exercise Test (SWEET), which is specific and individualized at the ventilatory threshold $[3,7,12]$.

\section{METHODS}

\section{Subjects}

Fourteen patients undergoing THA volunteered for the study. Their physical characteristics were (mean \pm standard deviation): age $75.1 \pm 4.8$, mass $73.8 \pm 13.5 \mathrm{~kg}$, height $158.2 \pm 7.9 \mathrm{~cm}$, and body mass index $29.3 \pm$ $4.7 \mathrm{~kg} / \mathrm{m}^{2}$. The detailed description of our patients and the rehabilitation program was recently published [7-9]. Briefly, only volunteers over the age of 65 whose main diagnosis was primary hip OA were included. Patients were excluded if they were undergoing revision arthroplasties, were unable to provide the informed consent, had medication that might have interfered with exercise testing and training, or presented pathologies such as decompensated congestive heart failure, acute myocarditis, acute myocardial infarction, unstable angina pectoris, uncontrolled cardiac arrhythmias, severe aortic stenosis, severe hypertension, and untreated hypertrophic obstructive cardiomyopathy. We used the Mini-Mental State Examination (MMSE) to evaluate the cognitive state of patients [10]. The patients were recruited as a convenient sample of volunteers of patients attending the hospital Polyclinique de Franche-Comté in Besançon, France. The study plan was accepted by the local ethical committee, and all patients signed an informed consent form.

\section{Procedures}

After surgery, patients were randomly assigned to one of the two groups: a training group (TG) $(n=7,6$ females and 1 male) or a control group (CG) ( $n=7,6$ females and 1 male). Both groups stayed at the rehabilitation center for 6 weeks, participating in a traditional daily rehabilitation program that consisted of exercises for muscular strength, range of motion, aquatics, and walking for 2 hours a day. In addition to the traditional rehabilitation program, TG undertook an exercise training program with an arm ergometer.

All patients performed an incremental exercise test until exhaustion on an arm-crank ergometer (Monark Rehab Trainer, Model 881E, Vansbro, Sweden) 1 month before THA, as described elsewhere [7]. Patients performed the test on a mechanically braked arm-crank ergometer in a fully upright sitting position. Respiratory gas exchange was measured with a portable metabolic measurement system cart (Aerosport KB1-C, Aerosport Inc, Ann Arbor, Michigan). Patients also performed a 6 min walking test at 2 months and 1 year after surgery. A condition-specific WOMAC OA Index (Likert format) was also completed 1 month before and 2 months and 1 year after surgery. WOMAC was presented in Likert format with 24 questions that are divided into 3 dimensions: pain (5 items), stiffness (2 items), and physical function (17 items) [11]. A high score on the WOMAC questionnaire indicates that the patient perceived a low personal health status.

The arm exercise program consisted of three sessions a week for 6 weeks of $30 \mathrm{~min}$ of the SWEET program proposed by Gimenez et al. [12]. One exercise session consisted of six consecutive periods of $5 \mathrm{~min}$, including 4 min of "base" work followed by 1 min of "peak" work. Initially, we set the base at the ventilatory threshold and the peak at the maximum tolerated power that were determined during the progressive test using the corresponding target heart rate. To maintain a sufficient intensity during training (target heart rate) to reach a peak heart rate by the end of sixth peak, we alternately increased the loads for the base and peak ( $5 \mathrm{~W}$ ). This readjustment was made when the heart rate at the end of the exercise was lowered by 10 to 12 beats per minute compared with the previous session. 
At 2 months and 1 year, both TG and CG performed a 6 min walking test [13]. The walking track was a $30 \mathrm{~m}-$ long empty hospital corridor. Patients were asked to walk back and forth along the track and to cover the longest possible distance in $6 \mathrm{~min}$. Unfortunately, four subjects, two from the TG and two from the CG, refused to perform the walking test 1 year after surgery.

\section{Statistics}

Results are presented in the Table as median scores, with extreme values in parentheses. Data were analyzed with the Mann-Whitney test for group comparisons and Wilcoxon signed-rank test for paired comparisons. The level of statistical significance was set at $p<0.05$.

\section{RESULTS}

All the patients obtained an MMSE score above 24, indicating they did not present any cognitive impairment and could understand each indication and provide an informed consent. Results are summarized in the Table. The TG covered a significantly longer distance than the CG in the 6 min walking test at 2 months (mean values of 396 vs $268 \mathrm{~m}$, respectively, $p<0.05$ ) and also at 1 year after surgery (mean values of 494 vs $406 \mathrm{~m}$, respectively, $p<0.05$ ). However, both groups improved their performance in the walking test at 1 year compared with the performance at 2 months $(p<0.05)$. The difference between the groups in the distance walked in the $6 \mathrm{~min}$ walking test was considerably reduced 1 year after surgery compared with the one observed at 2 months, but this difference was still statistically significant $(p<0.05)$.
Before surgery, both groups presented similar WOMAC scores (the median scores were 23 vs 27 for the physical functions aspect of the WOMAC and 36 vs 41 for the global score in TG and CG, respectively). Both groups significantly improved all dimensions of WOMAC at 2 months and 1 year after surgery compared with presurgery results $(p<0.05)$. However, TG obtained significantly lower WOMAC scores 2 months and 1 year after surgery compared with CG $(p<0.05)$.

\section{DISCUSSION}

After THA, traditional postoperative rehabilitation (the usual physiotherapy) may not promote patients' maximum possible recovery. The low level of daily physical activities performed by patients after THA may lead to deterioration in both exercise capacity and physical condition [14]. Such a situation has strong negative influences on the patient's functioning, health status, and quality of life.

During the 2-month period after THA, the only difference in treatment between our two groups of elderly patients was the interval-training program with the upper limbs (6 weeks, 3 times a week, 30 min each session). We used an interval-training program known to be effective to improve cardiorespiratory fitness. This program, SWEET, is specific and individualized at the ventilatory threshold [12]. Only an exercise program for the upper limbs was considered. Although patients would have been able to walk a few days after surgery, they would not have been able to perform training that would be strenuous

Table.

Changes in Western Ontario and McMaster Universities (WOMAC) physical function aspects and global score and results of walking test 1 month before, 2 months after, and 1 year after surgery in training group (TG) and control group (CG).

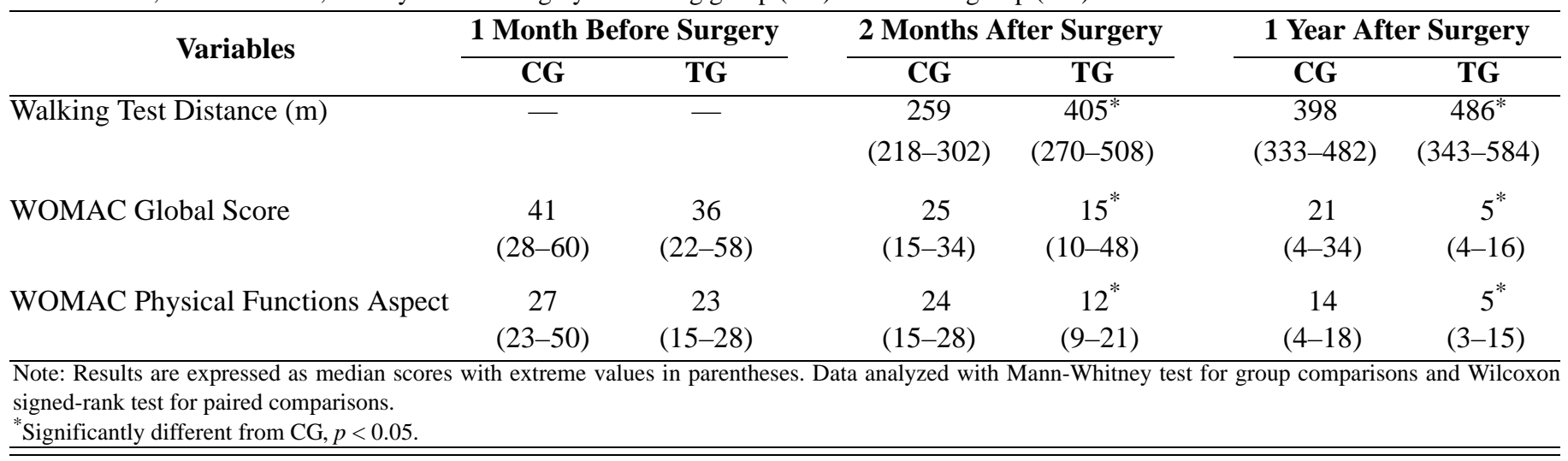


enough to promote an improvement in cardiorespiratory fitness. Such training with the lower limbs might be more appropriate a few weeks or months after arthroplasty. Important to note is that all our patients from both programs finished the 6-week rehabilitation period without complications. Our training seemed suitable for elderly OA patients and was clinically well tolerated (e.g., no injuries were reported). During the 1 year follow-up, no major health problems were reported in TG. Unfortunately, one case of phlebitis and one case of pulmonary edema were observed in CG. Those two subjects did not complete the 1-year follow-up.

Altogether, our interval-training program was able to induce an improvement in upper-body aerobic capacity, walking ability, and subjective health status (WOMAC score) [7-9]. In the walking tests, the TG covered a longer walking distance with a faster walking speed and with a longer stride length, and the cadence was higher (data not shown). Therefore, besides an improvement in fitness, the TG might also have improved in gait efficiency. One could speculate that the improvements induced by our upper-body exercise training could have stemmed from systemic cardiovascular and cardiorespiratory effects (central adaptations) rather than localized metabolic or haemodynamic changes. Upper-limb exercise has been reported to induce stronger cardiovascular stimuli for a given level of submaximal work than does lower-limb exercise [15]. This may explain, in part, our observed improvements in the incremental exercise tests and in the walking test. However, because of the impressive improvement in the walking capacities of our trained patients compared with their matched controls, especially 2 months after the surgery, we can suggest that some transfer effects had occurred. Such effects have previously been reported in endurance training with lower limbs where some improvements in exercise capacity of the upper limbs (untrained muscle group) were observed and vice versa [16]. Improved perception of the patients' physical possibilities and positive psychological effects of the training may also have had a positive impact.

The limitation of our study is the small number of subjects. Important characteristics of subjects (e.g., trainability) may possibly not have been evenly distributed between the two groups, even with random assignment of subjects to the groups. Therefore, selection bias may possibly explain, in part, the large functional improvements in the trained subjects. Moreover, because of pain and the patients' difficulties with movement, organizing a walking test before THA was, unfortunately, difficult. Also, our conclusions might mainly apply to female elderly patients, because we had only one male volunteer in each group. However, the differences observed in the walking ability between the groups were still of such magnitude, especially 2 months after surgery, that further studies are warranted in this area.

\section{CONCLUSIONS}

Although surgery had a major impact on our patients' physical fitness and functional status, the beneficial effects of upper-body physical training were statistically significant on those outcomes 2 months and 1 year after surgery. Such results, if confirmed, are striking and should be considered for improving the current practices in rehabilitation after such surgery. Therefore, a larger randomized controlled trial should be organized before the present results can be fully accepted into evidenced-based practice.

\section{ACKNOWLEDGMENTS}

We thank the volunteers who participated in our study. The material is based on work supported by the Association Nationale pour la Formation Hospitalière de FrancheCompté that supported J. Maire during his leave from the University Hospital of Besançon, grant 003/2000/1.

The authors have declared that no competing interests exist.

\section{REFERENCES}

1. Lonsdorfer J, Lampert E, Mettauer B, Charloux A, Geny B, Oswald M, Lonsdorfer-Wolfe E. Physical training in heart transplant recipients. Physiological aspects. Med Sport Sci. 1998;42:45-66.

2. Cherubini A, Donahue JL, Lowenthal DT, Mecocci P, Meuleman JR, Maggio D, Senin U. The effects of aging and endurance exercise on cardiovascular performance in healthy elderly: A review of the literature. Am J Geriatr Cardiol. 1998;7(2):25-32. [PMID: 11416449]

3. Maire J, Tordi N, Parratte B, Lonsdorfer J, Rouillon JD. Cardiovascular deconditioning in elderly with hip osteoarthrosis and benefits of reconditioning program after total hip arthroplasty. Sci Sports. 2002;17(4):155-65.

4. Wang AW, Gilbey HJ, Ackland TR. Perioperative exercise programs improve early return of ambulatory function after total hip arthroplasty: A randomized, controlled trial. Am J Phys Med Rehabil. 2002;81(11):801-6. [PMID: 12394990] 
5. Trudelle-Jackson E, Smith S. Effects of a late-phase exercise program after total hip arthroplasty: A randomized controlled trial. Arch Phys Med Rehabil. 2004;85(7):1056-62. [PMID: 15241750$]$

6. Hesse S, Werner C, Seibel H, Von Frankenberg S, Kappel EM, Kirker S, Kading M. Treadmill training with partial body-weight support after total hip arthroplasty: A randomized controlled trial. Arch Phys Med Rehabil. 2003;84(12): 1767-73. [PMID: 14669181]

7. Maire J, Dugué B, Faillenet-Maire AF, Tordi N, Parratte B, Smolander J, Rouillon JD. Recovery after total hip joint arthroplasty in elderly patients with osteoarthritis: Positive effect of upper limb interval-training. J Rehabil Med. 2003; 35(4):174-79. [PMID: 12892243]

8. Maire J, Faillenet-Maire AF, Grange CC, Dugué B, Tordi N, Parratte B, Rouillon JD. A specific arm-interval exercise program could improve the health status and walking ability of elderly patients after total hip arthroplasty: A pilot study. J Rehabil Med. 2004;36(2):92-94. [PMID: 15180224]

9. Grange CC, Maire J, Groslambert A, Tordi N, Dugué B, Pernin JN, Rouillon JD. Perceived exertion and rehabilitation with arm crank in elderly patients after total hip arthroplasty: A preliminary study. J Rehabil Res Dev. 2004; 41(4):611-20. [PMID: 15558389]

10. Folstein MF, Folstein SE, McHugh PR. "Mini-mental state.” A practical method for grading the cognitive state of patients for the clinician. J Psychiatr Res. 1975;12(3):189-98. [PMID: 1202204$]$
11. Roos EM, Roos HP, Lohmander LS. WOMAC Osteoarthritis Index-Additional dimensions for use in subjects with posttraumatic osteoarthritis of the knee. Western Ontario and MacMaster Universities. Osteoarthritis Cartilage. 1999;7(2): 216-21. [PMID: 10222220]

12. Gimenez M, Predine E, Marchand M, Servera E, Ponz JL, Polu JM. Implications of lower- and upper-limb training procedures in patients with chronic airway obstruction. Chest. 1992;101(5 Suppl):279S-88S. [PMID: 1576850]

13. Harada ND, Chiu V, Stewart AL. Mobility-related function in older adults: Assessment with a 6-minute walk test. Arch Phys Med Rehabil. 1999;80(7):837-41. [PMID: 10414771]

14. Morlock M, Schneider E, Bluhm A, Vollmer M, Bergmann G, Muller V, Honl M. Duration and frequency of every day activities in total hip patients. J Biomech. 2001;34(7):873-81. [PMID: 11410171$]$

15. Walker RD, Nawaz S, Wilkinson CH, Saxton JM, Pockley AG, Wood RF. Influence of upper- and lower-limb exercise training on cardiovascular function and walking distances in patients with intermittent claudication. J Vasc Surg. 2000;31(4):662-69. [PMID: 10753273]

16. Tordi N, Belli A, Mougin F, Rouillon JD, Gimenez M. Specific and transfer effects induced by arm or leg training. Int J Sports Med. 2001;22(7):517-24. [PMID: 11590479]

Submitted for publication March 4, 2005. Accepted in revised form January 23, 2006. 